\title{
Comparison of the quality of total mesorectal excision after robotic and laparoscopic surgery for rectal cancer: a multicenter, propensity score-matched study
}

\author{
Keehyun Park', Sohyun Kim², Hye Won Lee ${ }^{3}$, Sung Uk Bae', Seong Kyu Baek', Woon Kyung Jeong ${ }^{1}$ \\ 'Department of Surgery, Keimyung University Dongsan Medical Center, Daegu; \\ 2Department of Surgery, Yeungnam University Medical Center, Daegu; \\ ${ }^{3}$ Department of Pathology, Keimyung University Dongsan Medical Center, Daegu, Korea
}

Purpose: This study aimed to evaluate and compare the quality of total mesorectal excision (TME) and disease-free and overall survival rates between robotic and laparoscopic surgeries for rectal cancer.

Methods: From January 2015 to December 2018, 234 patients underwent curative robotic or laparoscopic surgery for rectal cancer at two centers. Ultimately, 201 patients were enrolled. To control for different demographic factors in the two groups, propensity score matching was used at a 1:1 ratio. Propensity scores were generated with the baseline characteristics, including age, sex, body mass index, American Society of Anesthesiologists score, previous abdominal surgery, tumor location, preoperative chemotherapy, and preoperative radiation. Finally, 134 patients were matched with 67 patients in the robotic surgery group and 67 patients in the laparoscopic surgery group.

Results: There was no significant difference in the pathologic stages between the robotic and laparoscopic surgery groups. Distal margin involvement was only observed in the robotic surgery group $(1 / 67,1.5 \%)$. Circumferential resection margin involvement was not different between the robotic surgery and laparoscopic surgery groups (3/67 [4.5\%] and 4/67 [6.0\%], respectively, $P=1.000$ ). The quality of TME (complete, nearly complete, and incomplete) was similar between the robotic surgery and laparoscopic surgery groups $(88.0 \%, 6.0 \%, 6.0 \%$ and $79.1 \%, 9.0 \%, 11.9 \%$, respectively, $\mathrm{P}=0.358$ ). The disease-free and overall survival rates were not significantly different between the groups. Conclusion: The quality of TME and disease-free and overall survival rates between the two surgeries were similar. There was no oncologic advantage of robotic surgery for rectal cancer compared to laparoscopic surgery.

Keywords: Rectal cancer, Robotic surgery, Total mesorectal excision, Survival, Recurrence

Received: Aug 15, 2021 Revised: Nov 30, 2021 Accepted: Dec 8, 2021 Correspondence to: Woon Kyung Jeong

Department of Surgery, Keimyung University Dongsan Medical Center, 1035 Dalgubeol-daero, Dalseo-gu, Daegu 42601, Korea

Tel: +82-53-258-7879, Fax: +82-53-258-4710

E-mail: shinycloud@dsmc.or.kr

ORCID: Keehyun Park (https://orcid.org/0000-0003-2089-3267), Sohyun Kim (https://orcid.org/0000-0002-8625-329X), Hye Won Lee (https://orcid.org/00000001-8540-524X), Sung Uk Bae (https://orcid.org/0000-0002-7876-4196), Seong Kyu Baek (https://orcid.org/0000-0001-6427-8675), Woon Kyung Jeong (https:// orcid.org/0000-0001-8421-218X)

Copyright @ 2021 Korean Society of Surgical Oncology

This is an Open Access article distributed under the terms of the Creative Commons Attribution Non-Commercial License (http://creativecommons.org/licenses/by-nc/4.0) which permits unrestricted non-commercial use, distribution, and reproduction in any medium, provided the original work is properly cited.

\section{INTRODUCTION}

In rectal cancer, total mesorectal excision (TME) is a necessary procedure for reducing local recurrence. The mesorectum is the adipose and lymphatic tissues encircling the rectum. The TME procedure sharply dissects the avascular plane between the parietal fascia and the visceral fascia covering the mesorectum. In 1982, Heald et al. [1] suggested the concept of TME to reduce postoperative local recurrence. A local recurrence of $2.6 \%$ in rectal cancer after TME was reported in 1986 [2]. Subsequently, numerous studies reported a low local recurrence after TME of 3\%-11\%, comparable with the original operative method in which local recurrence reached 38\% [3]. However, local recurrence after TME was very different in each study, and there was over $10 \%$ local recurrence af- 
ter TME in some studies. The possibility of inappropriate TME may have caused different local recurrence rates to emerge. In a study that classified TME quality into three grades (complete, nearly complete, and incomplete), the incomplete TME patient group showed a statistically higher local/distant recurrence rate than the complete TME patient group [4]. As many studies report, the better the quality of TME, the better the oncological outcome.

Recently, robotic surgery (RS) has been used as a choice for rectal cancer surgery. Compared with the original laparoscopic surgery (LS), RS has the advantage that delicate dissection can be performed in the narrow pelvic cavity by using multiple joints. However, several studies that compared RS with LS showed conflicting results in terms of postoperative outcomes. Some studies have reported that RS in rectal cancer has better sexual/urinary function, less blood loss, and faster recovery [5-7]. On the other hand, some studies have reported that despite the high cost of RS, oncological outcomes are not better than LS for rectal cancer [8,9] . Most of all, few studies have compared robotic and laparoscopic surgeries in terms of TME quality, which can affect postoperative oncological outcomes, and every study enrolled a small number of patients and was performed retrospectively in a single center [10]. Therefore, we designed a multicenter retrospective study to compare TME quality and long-term oncologic outcomes between RS and LS in rectal cancer by using propensity score matching to reduce possible bias.

\section{METHODS}

\section{Study population and design}

From January 2015 to December 2018, 234 patients underwent curative RS or LS for rectal cancer at two tertiary referral hospitals. Patient data were collected from a prospectively managed electronic database. Thirty-three patients who underwent simultaneous multiple organ resections combined with rectal cancer, had stage IV rectal cancer, or other cancers were excluded.

Ultimately, 201 patients were enrolled in the study. The quality of TME was assessed by a pathologist at each center. To control for different demographic factors in the two groups, propensity score matching was used at a 1:1 ratio. Propensity scores were generated with the baseline characteristics, including age, sex, body mass index, American Society of Anesthesiologists score, previous abdominal surgery, tumor location, preoperative chemotherapy, and preoperative radiation. Finally, 134 patients were matched with 67 patients in the RS group and 67 patients in the LS group (Fig. 1).

\section{Statistical analysis}

The data are displayed as frequencies and percentages for categori-

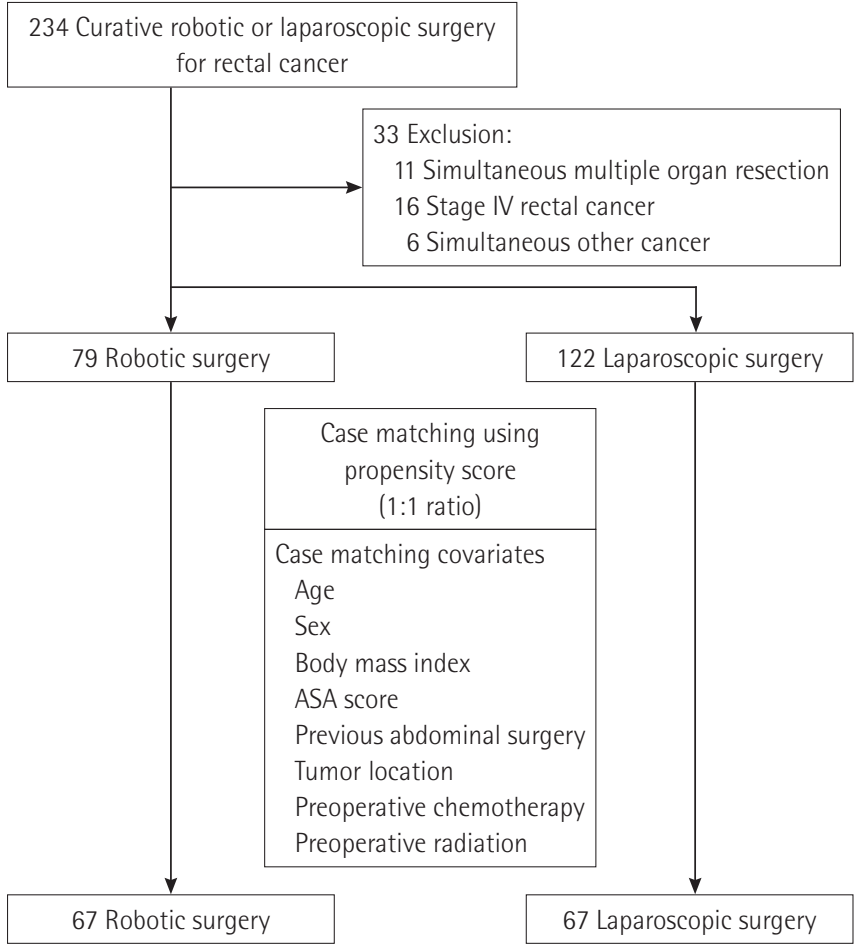

Fig. 1. Study design. ASA, American Society of Anesthesiologists.

cal variables and were analyzed using Pearson chi-square test or Fisher exact test. Kolmogorov-Smirnov test was used to assess the distribution of continuous variables. Normally distributed variables were examined using the Student $\mathrm{t}$-test, and the results are presented as means (standard deviations). Conversely, non-normally distributed continuous variables were analyzed using the Mann-Whitney $\mathrm{U}$ test, and the results are expressed as medians (interquartile ranges). Kaplan-Meier method was used to analyze survival. Statistical analyses were performed using PASW Statistics 18 software (SPSS Inc., Chicago, IL, USA) and R 3.2.2 (The R Project for Statistical Computing, Vienna, Austria). Two-tailed values of $\mathrm{P}<0.05$ were considered statistically significant.

\section{Ethics statement}

The study protocol was reviewed and approved by the Institutional Review Board (IRB) of the Keimyung University Dongsan Hospital (IRB No. 2019-06-001). The need for informed consent was waived owing to the retrospective study design.

\section{RESULTS}

Demographic and preoperative data before and after propensity score matching

Before propensity score case matching, the RS group had a lower mean age, higher male percentage, higher preoperative chemo- 
therapy rate, and higher preoperative radiation rate than the LS group. Patient's mean ages were 62.8 years and 68.3 years in the RS and LS groups, respectively $(\mathrm{P}<0.01)$. The proportions of men were $69.6 \%$ and $53.3 \%$ in in the RS and LS groups, respectively $(\mathrm{P}=0.021)$. The preoperative chemoradiotherapy rates were $55.7 \%$ and $18.0 \%$ in the RS and LS groups, respectively $(\mathrm{P}<0.01)$.

After propensity score case matching, those aforementioned factors became well balanced between the groups. Patients' mean ages were 63.1 years and 66.1 years in the RS and LS groups, respectively $(\mathrm{P}=0.086)$. The male percentages were similar between the RS and LS groups (64.2\% and 61.2\%, respectively, $\mathrm{P}=0.721$ ). Moreover, preoperative chemoradiotherapy rates were not statistically different between the groups ( $47.8 \%$ and $29.9 \%$, respectively; $\mathrm{P}=0.051)$ (Table 1).

\section{Perioperative results after propensity score matching}

Although the operative time was longer in the RS group than in the LS group, a statistical difference was not observed (224.8 minutes and 195.8 minutes, respectively, $\mathrm{P}=0.530$ ) (Table 2). When comparing TME time between the groups, the RS group showed a shorter TME time than the LS group. However, there was no statistical difference (65.4 minutes and 68.4 minutes, respectively, $\mathrm{P}=$ 0.847). Although conversion to open laparotomy was required only in the LS group, the difference was not statistically significant ( 0 and 2 cases, respectively, $\mathrm{P}=0.496$ ). There were no statistical differences in the diverting stoma rate, first flatus, first soft diet, and length of stay between the groups.

The postoperative complication rate was higher in the LS group than in the RS group; however, the difference was not statistically significant (19/67 [28.4\%] and 30/67 [44.8\%], respectively, $\mathrm{P}=0.720$ ). The Clavien-Dindo grade was also not significantly different between the groups. Most complications were classified as Clavien-Dindo grade I or II in both groups. Grade IIIb complications occurred in three patients in the RS group. All three patients experienced anastomosis leakage requiring transanal sutures with or without diverting ileostomy. One patient in the LS group had a grade IIIb complication and underwent transanal suture to treat anastomosis bleeding.

\section{Pathologic results after propensity score matching}

The quality of TME (complete, nearly complete, and incomplete) was similar between the RS and LS groups $(88.0 \%, 6.0 \%, 6.0 \%$ and 79.1\%, 9.0\%, 11.9\%, respectively, $\mathrm{P}=0.358$ ) (Table 3). Pathologic stages including the (y)pT stage, (y)pN stage, and (y)p stage were similar between the groups. Other pathologic data, including differentiation, lymphovascular invasion, and perineural invasion, were not significantly different between the groups. The mean number of retrieved lymph nodes was not significantly different between the groups (13.6 and 13.1, respectively, $\mathrm{P}=0.686$ ). Circumferential resection margin involvement was not different between the RS and LS groups (3/67 [4.5\%] and 4/67 [6.0\%], respectively, $\mathrm{P}=1.000$ ). Although a positive distal margin was observed

Table 1. Preoperative factors (before and after case matching)

\begin{tabular}{|c|c|c|c|c|c|c|}
\hline \multirow[b]{2}{*}{ Variable } & \multicolumn{3}{|c|}{ Before matching } & \multicolumn{3}{|c|}{ After matching } \\
\hline & $\begin{array}{l}\text { Robotic } \\
(n=79)\end{array}$ & $\begin{array}{l}\text { Laparoscopic } \\
\qquad(n=122)\end{array}$ & P-value & $\begin{array}{l}\text { Robotic } \\
(n=67)\end{array}$ & $\begin{array}{l}\text { Laparoscopic } \\
\qquad(n=67)\end{array}$ & P-value \\
\hline Age $(y r)$, mean $\pm S D$ & $62.8 \pm 10.4$ & $68.3 \pm 9.8$ & $<0.01$ & $63.1 \pm 10.6$ & $66.1 \pm 9.9$ & 0.086 \\
\hline Sex, No. $(\%)$ & & & 0.021 & & & 0.721 \\
\hline Male & $55(69.6)$ & $65(53.3)$ & & $43(64.2)$ & 41 (61.2) & \\
\hline Female & $24(30.4)$ & $57(46.7)$ & & $24(35.8)$ & $26(38.8)$ & \\
\hline $\mathrm{BMI}\left(\mathrm{kg} / \mathrm{m}^{2}\right)$, mean $\pm \mathrm{SD}$ & $23.6 \pm 3.3$ & $23.9 \pm 4.0$ & 0.591 & $23.6 \pm 3.3$ & $23.2 \pm 4.1$ & 0.551 \\
\hline ASA score, №. (\%) & & & 0.953 & & & 0.867 \\
\hline 1 & $28(35.4)$ & $41(33.6)$ & & $25(37.3)$ & $24(35.8)$ & \\
\hline$\|$ & $41(51.9)$ & $66(54.1)$ & & $35(52.2)$ & $34(50.7)$ & \\
\hline III & $10(12.7)$ & $15(12.3)$ & & 7 (10.4) & $9(13.4)$ & \\
\hline Previous abdominal surgery, №. (\%) & $14(17.7)$ & $18(14.8)$ & 0.574 & $12(17.9)$ & $12(17.9)$ & 1.000 \\
\hline Tumor location, №. (\%) & & & 0.124 & & & 0.214 \\
\hline Upper ( $\geq 11 \mathrm{~cm})$ & $7(8.9)$ & $3(2.5)$ & & $7(10.4)$ & $2(3.0)$ & \\
\hline Middle $(6-10 \mathrm{~cm})$ & $44(55.7)$ & $74(60.7)$ & & $38(56.7)$ & $43(64.2)$ & \\
\hline Lower $(\leq 5 \mathrm{~cm})$ & $28(35.4)$ & 45 (36.9) & & $22(32.8)$ & $22(32.8)$ & \\
\hline Preoperative chemoradiotherapy, №. (\%) & $44(55.7)$ & $22(18.0)$ & $<0.01$ & $32(47.8)$ & $20(29.9)$ & $0.051^{\mathrm{a}}$ \\
\hline
\end{tabular}

SD, standard deviation; BMI, body mass index; ASA, American Society of Anesthesiologists.

a) Fisher exact test. 
Table 2. Perioperative results (after case matching)

\begin{tabular}{|c|c|c|c|}
\hline Variable & Robotic $(n=67)$ & Laparoscopic $(n=67)$ & P-value \\
\hline Operation time (min), mean \pm SD & $224.8 \pm 99.4$ & $195.8 \pm 70.1$ & 0.530 \\
\hline TME time (min), mean \pm SD & $65.4 \pm 52.9$ & $68.4 \pm 58.8$ & 0.847 \\
\hline Conversion to open laparotomy, №. (\%) & 0 & $2(3.0)$ & 0.496 \\
\hline Diverting stoma, №. (\%) & $39(58.2)$ & $39(58.2)$ & 1.000 \\
\hline First flatus (day), mean $\pm S D$ & $2.3 \pm 1.4$ & $2.6 \pm 1.8$ & 0.229 \\
\hline First soft diet (day), mean \pm SD & $6.0 \pm 2.7$ & $6.2 \pm 2.7$ & 0.622 \\
\hline LOS (day), mean \pm SD & $10.3 \pm 3.9$ & $11.4 \pm 3.7$ & 0.280 \\
\hline Morbidity (30 days), No. (\%) & 19 (28.4) & $30(44.8)$ & 0.720 \\
\hline SSI (superficial) & 5 & 7 & \\
\hline SSI (deep) & 0 & 2 & \\
\hline SSI (organ/space) & 3 & 11 & \\
\hline Anastomosis leakage & 2 & 0 & \\
\hline Pseudomembranous colitis & 2 & 1 & \\
\hline Postoperative bleeding & 0 & 1 & \\
\hline Urinary retention & 1 & 2 & \\
\hline Urinary tract infection & 0 & 1 & \\
\hline Chyle leakage & 3 & 0 & \\
\hline Small intestine ileus & 2 & 5 & \\
\hline Heart complication & 1 & 0 & \\
\hline Subcutaneous emphysema & 0 & 1 & \\
\hline Clavien-Dindo classification, №. (\%) & & & 0.062 \\
\hline 1 & $10(52.6)$ & 10 (33.3) & \\
\hline$\|$ & $6(31.6)$ & $19(63.3)$ & \\
\hline$\| l b$ & $3(15.8)$ & $1(3.3)$ & \\
\hline Mortality (30 days), №. (\%) & 0 & 0 & \\
\hline
\end{tabular}

SD, standard deviation; TME, total mesorectal excision; LOS, length of stay; SSI, surgical site infection.

only in the RS group, there was no statistical difference between the RS and LS groups ( 1 case and 0 cases, respectively, $\mathrm{P}=1.000$ ). The total recurrence rate between the RS and LS groups was not significantly different ( $16.4 \%$ and $20.9 \%$, respectively, $\mathrm{P}=0.506$ ).

\section{Disease-free and overall survival rates}

Survival analysis between the RS and LS groups is shown in Fig. 2. The 5-year overall survival and disease-free survival rates were not significantly different between the groups. The 5 -year overall survival rates were $90.5 \%$ and $85.4 \%$ in the RS and LS groups, respectively $(\mathrm{P}=0.411)$. Meanwhile, the 5 -year disease-free survival rate was longer in the RS group, but the difference was not statistically significant between the groups ( $82.0 \%$ and $71.5 \%$, respectively, $\mathrm{P}=0.098)$.

\section{DISCUSSION}

It is obvious that performing adequate TME is necessary to reduce local recurrence. Surgeons need a better view and to perform deli- cate movements during rectal cancer surgery to improve the quality of TME. Surgical robots can overcome the limitations of LS through better dexterity, articulated arm, a three-dimensional view, magnification, elimination of tremors, and a stable camera. These advantages can provide precise dissection, better identification, and preservation of small structures in the pelvic cavity [1114]. In this regard, RS for rectal cancer is expected to increase the quality of TME. Accordingly, better long-term oncologic outcomes are expected. However, the clinical benefits of RS for rectal cancer have not yet been confirmed.

RS had been expected to have advantages over the LS for rectal cancer. This study, however, showed that there were no significant differences in TME quality, recurrence rates, and disease-free and overall survival rates between robotic and laparoscopic surgeries for rectal cancer. Many previous studies also showed no differences in long-term oncologic outcomes between robotic and laparoscopic surgeries $[12,15,16]$. However, some studies have shown that disease-free and overall survival rates increase after RS for rectal cancer $[17,18]$. Most studies enrolled a small number of patients 
Table 3. Pathologic results (after case matching)

\begin{tabular}{|c|c|c|c|}
\hline Variable & Robotic $(n=67)$, No. $(\%)$ & Laparoscopic $(n=67)$, No. $(\%)$ & P-value \\
\hline TME grading & & & 0.358 \\
\hline Complete & $59(88.0)$ & $53(79.1)$ & \\
\hline Nearly complete & $4(6.0)$ & $6(9.0)$ & \\
\hline Incomplete & $4(6.0)$ & $8(11.9)$ & \\
\hline (y)pT stage & & & 0.436 \\
\hline CR & $1(1.5)$ & $2(3.0)$ & \\
\hline Tis, T1, T2 & $44(65.7)$ & $37(55.2)$ & \\
\hline $\mathrm{T} 3, \mathrm{~T} 4$ & $22(32.8)$ & $28(41.8)$ & \\
\hline (y)pN stage & & & 0.150 \\
\hline No & $51(76.1)$ & $41(61.2)$ & \\
\hline N1 & $11(16.4)$ & $20(29.9)$ & \\
\hline N2 & $5(7.5)$ & $6(9.0)$ & \\
\hline (y)pStage & & & 0.296 \\
\hline $\mathrm{pCR}$ & $1(1.5)$ & $2(3.0)$ & \\
\hline 0 & $1(1.5)$ & $1(1.5)$ & \\
\hline । & 33 (49.3) & $22(32.8)$ & \\
\hline$\|$ & $16(23.9)$ & $16(23.9)$ & \\
\hline III & $16(23.9)$ & $26(38.8)$ & \\
\hline Differentiation & & & 0.361 \\
\hline WD & $6(9.0)$ & $4(6.0)$ & \\
\hline MD & $58(86.6)$ & $56(83.6)$ & \\
\hline PD, mucinous & $3(4.5)$ & $7(10.4)$ & \\
\hline Lymphovascular invasion & & & 0.190 \\
\hline Negative & $57(85.1)$ & $51(76.1)$ & \\
\hline Positive & $10(14.9)$ & $16(23.9)$ & \\
\hline Perineural invasion & & & 0.235 \\
\hline Negative & $59(88.1)$ & $54(80.6))$ & \\
\hline Positive & 8 (11.9) & $13(19.4)$ & \\
\hline Retrieved lymph node, mean \pm SD & $13.6 \pm 5.4$ & $13.1 \pm 5.3$ & 0.686 \\
\hline Positive CRM ( $\leq 1 \mathrm{~mm})$ & $3(4.5)$ & $4(6.0)$ & $1.000^{\mathrm{a})}$ \\
\hline Positive distal margin & $1(1.5)$ & 0 & $1.000^{\mathrm{a})}$ \\
\hline Recurrence & $11(16.4)$ & $14(20.9)$ & 0.506 \\
\hline Loco-regional & 2 & 4 & \\
\hline Systemic & 9 & 10 & \\
\hline
\end{tabular}

TME, total mesorectal excision; $C R$, complete remission; $P C R$, pathologic complete remission; WD, well differentiated; $M D$, moderately differentiated; $P D$, poorly differentiated; SD, standard deviation; CRM, circumferential resection margin.

a) Fisher exact test.

to evaluate the superiority of RS. Hence, it remains controversial whether long-term oncologic outcomes of RS are better than those of LS for rectal cancer.

High-risk conditions may cause poor vision of the operative field, including the male pelvic cavity, which is deeper and narrower than that of women, obesity, and low-lying rectal cancer. RS may provide some benefits to patients with these conditions. Several studies have reported low open conversion rates in robotic rectal cancer surgery in patients with these conditions $[8,16]$. Nevertheless, there is currently no consensus about which patients with rec- tal cancer can benefit most from RS. Further studies are necessary to answer this question.

In the present study, the quality of TME was similar between the two groups. However, some studies have shown better quality of TME in the RS group $[10,19]$. This difference in the results among the studies may be due to the different proportions of the tumor location in the studies. To adequately compare the quality of TME, only mid or low rectal cancer should be included. Surgery for upper rectal cancer does not require deep dissection of the pelvis. Hence, achieving complete quality of TME is much easier. In the 

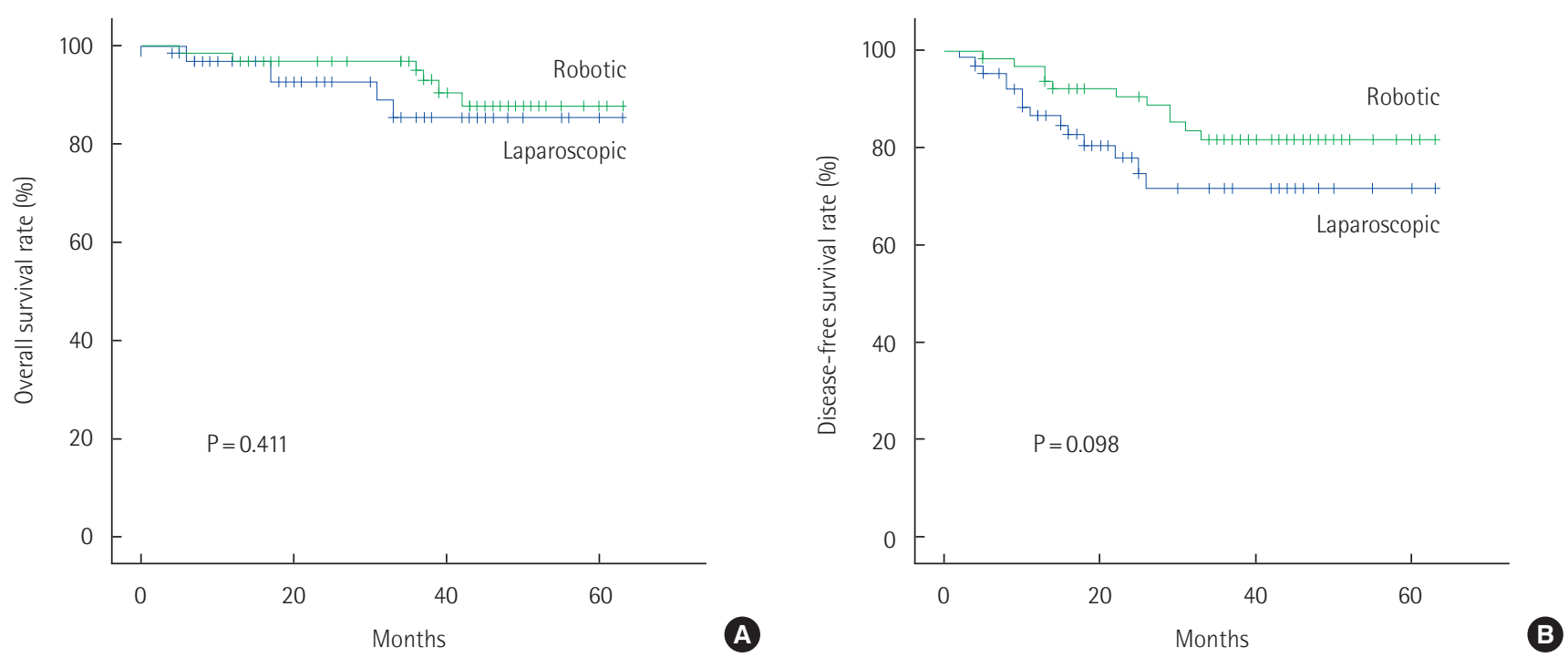

Fig. 2. Five-year overall survival curves (A) and disease-free survival curves (B) between robotic and laparoscopic surgery groups.

current study, tumor location was not different between the two groups, and the proportion of patients with low rectal cancer was the same. However, the proportion of patients with upper rectal cancer was higher in the RS group than in the LS group.

This study has some limitations. First, it was not a randomized, controlled, or prospective trial. To reduce selection bias, we used propensity score matching. Second, the number of patients enrolled in this study was too small to generalize the results. In future studies, a large number of patients should be enrolled to gain adequate study power. Third, there were potential biases due to different RS techniques and the different experiences of the participating surgeons. The surgical field of rectal cancer surgery is wide, spanning from the pelvic cavity to the splenic flexure. Therefore, various RS techniques have been developed for treating rectal cancer. Some surgeons prefer a hybrid technique consisting of a laparoscopic procedure for ligation of the inferior mesenteric vessels and mobilization of the splenic flexure and robotic procedures for pelvic dissection $[15,20]$. Other surgeons performed a multi-docking procedure by repositioning the operation bed and robotic arms $[21,22]$. In contrast, some robotic surgeries were completed using a single-docking procedure $[23,24]$. These differences in robotic surgical techniques can cause biases. Standardization of the RS technique is required to reduce such biases.

Fourth, many surgeons participated in this study. The learning curves of surgeons in robotic rectal surgery were different. This may have influenced the clinical or pathologic outcomes. Accordingly, the comparison between robotic and laparoscopic surgeries for rectal cancer is less accurate. Most surgeons were more familiar with LS than with RS. In contrast to robotic colon surgery introduced first in 2002 [25], laparoscopic colon surgery was first re- ported in 1991 [26]. Surgeons who do not have enough experience in RS are more likely to choose LS rather than RS. On the other hand, if surgeons are already in the plateau phase of the learning curve or have extensive experience with RS, they tend to use robotic systems for patients with advanced cancer or a narrow pelvic cavity or cancer located near the anus. At this point, a selection bias might have been made by the surgeon.

While surgeons go from the learning phase to the plateau phase during RS by performing the same procedures over and over, a better quality of TME can be achieved [27]. Therefore, for accurate comparison of the quality of TME or other outcomes between robotic and laparoscopic surgeries, we must compare the patients who underwent surgery by surgeons at the plateau phase of the learning curve for RS or LS. Some studies mentioned that the threshold to be an expert who is in the plateau phase for RS. The common ground in these studies was that all surgeons had to perform RS in at least 20 cases to become an expert, although the threshold varied from 20 to 39 cases $[20,28]$.

The last limitation of this study is that postoperative sexual or urinary function, which can influence quality of life, was not evaluated. Many studies have compared urinary and sexual function between robotic and laparoscopic surgeries. They found that preventing pelvic autonomic nerve injury is helpful for maintaining preoperative urinary and sexual function. The preservation of these two functions can lead to a better quality of life after surgery [29]. Many studies have shown better preservation of urinary and sexual function in patients who underwent RS for rectal cancer $[5,30]$. However, some studies reported that there was no meaningful difference in functional outcomes between the RS and LS groups [16]. 
This multicenter, case-control retrospective analysis showed that there were no significant differences in the quality of TME and disease-free and overall survival rates between the RS and LS groups. There was no oncologic advantage of RS for rectal cancer compared to LS. Although 30-day morbidity seemed to be higher in the LS group than in the RS group, the difference was not statistically significant. Large, multicenter, prospective studies are needed to validate the advantages of the RS system used for rectal cancer.

\section{CONFLICT OF INTEREST}

Sung Uk Bae is an editorial board member of the journal but was not involved in the peer reviewer selection, evaluation, or decision process of this article. No other potential conflicts of interest relevant to this article were reported.

\section{ACKNOWLEDGMENTS}

KP was the main contributor and the first author of this paper. WKJ planned and organized the paper; WKJ and SK extracted and analyzed the data; SUB, SKB, SK, and HWL contributed materials/analysis tools; $\mathrm{KP}$ and $\mathrm{WKJ}$ contributed to the writing of the manuscript; and WKJ proofread the paper.

\section{REFERENCES}

1. Heald RJ, Husband EM, Ryall RD. The mesorectum in rectal cancer surgery: the clue to pelvic recurrence? Br J Surg 1982;69:613-6.

2. Heald RJ, Ryall RD. Recurrence and survival after total mesorectal excision for rectal cancer. Lancet 1986;1:1479-82.

3. Bulow S, Christensen IJ, Harling H, Kronborg O, Fenger C, Nielsen $\mathrm{HJ}$, et al. Recurrence and survival after mesorectal excision for rectal cancer. Br J Surg 2003;90:974-80.

4. Nagtegaal ID, van de Velde CJ, van der Worp E, Kapiteijn E, Quirke P, van Krieken JH, et al. Macroscopic evaluation of rectal cancer resection specimen: clinical significance of the pathologist in quality control. J Clin Oncol 2002;20:1729-34.

5. Kim HJ, Choi GS, Park JS, Park SY, Yang CS, Lee HJ. The impact of robotic surgery on quality of life, urinary and sexual function following total mesorectal excision for rectal cancer: a propensity score-matched analysis with laparoscopic surgery. Colorectal Dis 2018;20:O103-13.

6. Liu WH, Yan PJ, Hu DP, Jin PH, Lv YC, Liu R, et al. Short-term outcomes of robotic versus laparoscopic total mesorectal excision for rectal cancer: a cohort study. Am Surg 2019;85:294-302.

7. Tang X, Wang Z, Wu X, Yang M, Wang D. Robotic versus laparo- scopic surgery for rectal cancer in male urogenital function preservation, a meta-analysis. World J Surg Oncol 2018;16:196.

8. Jayne D, Pigazzi A, Marshall H, Croft J, Corrigan N, Copeland J, et al. Effect of robotic-assisted vs conventional laparoscopic surgery on risk of conversion to open laparotomy among patients undergoing resection for rectal cancer: the ROLARR randomized clinical trial. JAMA 2017;318:1569-80.

9. Lee SH, Kim DH, Lim SW. Robotic versus laparoscopic intersphincteric resection for low rectal cancer: a systematic review and meta-analysis. Int J Colorectal Dis 2018;33:1741-53.

10. Aselmann H, Kersebaum JN, Bernsmeier A, Beckmann JH, Moller T, Egberts JH, et al. Robotic-assisted total mesorectal excision (TME) for rectal cancer results in a significantly higher quality of TME specimen compared to the laparoscopic approach-report of a single-center experience. Int J Colorectal Dis 2018;33:1575-81.

11. Choi GS, Park IJ, Kang BM, Lim KH, Jun SH. A novel approach of robotic-assisted anterior resection with transanal or transvaginal retrieval of the specimen for colorectal cancer. Surg Endosc 2009; 23:2831-5.

12. Kim NK, Kang J. Optimal total mesorectal excision for rectal cancer: the role of robotic surgery from an expert's view. J Korean Soc Coloproctol 2010;26:377-87.

13. Maeso S, Reza M, Mayol JA, Blasco JA, Guerra M, Andradas E, et al. Efficacy of the Da Vinci surgical system in abdominal surgery compared with that of laparoscopy: a systematic review and meta-analysis. Ann Surg 2010;252:254-62.

14. Pigazzi A, Garcia-Aguilar J. Robotic colorectal surgery: for whom and for what? Dis Colon Rectum 2010;53:969-70.

15. Park EJ, Cho MS, Baek SJ, Hur H, Min BS, Baik SH, et al. Longterm oncologic outcomes of robotic low anterior resection for rectal cancer: a comparative study with laparoscopic surgery. Ann Surg 2015;261:129-37.

16. Rouanet P, Bertrand MM, Jarlier M, Mourregot A, Traore D, Taoum C, et al. Robotic versus laparoscopic total mesorectal excision for sphincter-saving surgery: results of a single-center series of 400 consecutive patients and perspectives. Ann Surg Oncol 2018; 25:3572-9.

17. Kim J, Baek SJ, Kang DW, Roh YE, Lee JW, Kwak HD, et al. Robotic resection is a good prognostic factor in rectal cancer compared with laparoscopic resection: long-term survival analysis using propensity score matching. Dis Colon Rectum 2017;60:266-73.

18. Tejedor P, Sagias F, Flashman K, Lee YH, Naqvi S, Kandala N, et al. The impact of robotic total mesorectal excision on survival of patients with rectal cancer: a propensity matched analysis. Int J Colorectal Dis 2019;34:2081-9.

19. Bedirli A, Salman B, Yuksel O. Robotic versus laparoscopic resection for mid and low rectal cancers. JSLS 2016;20:e2015.00110. 
20. Kim HJ, Choi GS, Park JS, Park SY. Multidimensional analysis of the learning curve for robotic total mesorectal excision for rectal cancer: lessons from a single surgeon's experience. Dis Colon Rectum 2014:57:1066-74.

21. D’Annibale A, Pernazza G, Monsellato I, Pende V, Lucandri G, Mazzocchi P, et al. Total mesorectal excision: a comparison of oncological and functional outcomes between robotic and laparoscopic surgery for rectal cancer. Surg Endosc 2013;27:1887-95.

22. Kwak JM, Kim SH, Kim J, Son DN, Baek SJ, Cho JS. Robotic vs laparoscopic resection of rectal cancer: short-term outcomes of a case-control study. Dis Colon Rectum 2011;54:151-6.

23. Ahmed J, Siddiqi N, Khan L, Kuzu A, Parvaiz A. Standardized technique for single-docking robotic rectal surgery. Colorectal Dis 2016;18:O380-4.

24. Park YA, Kim JM, Kim SA, Min BS, Kim NK, Sohn SK, et al. Totally robotic surgery for rectal cancer: from splenic flexure to pelvic floor in one setup. Surg Endosc 2010;24:715-20.

25. Weber PA, Merola S, Wasielewski A, Ballantyne GH. Telerobotic-assisted laparoscopic right and sigmoid colectomies for benign disease. Dis Colon Rectum 2002;45:1689-96.

26. Jacobs M, Verdeja JC, Goldstein HS. Minimally invasive colon resection (laparoscopic colectomy). Surg Laparosc Endosc 1991;1: 144-50.

27. Gachabayov M, Kim SH, Jimenez-Rodriguez R, Kuo LJ, Cianchi F, Tulina I, et al. Impact of robotic learning curve on histopathology in rectal cancer: a pooled analysis. Surg Oncol 2020;34:121-5.

28. Jimenez-Rodriguez RM, Rubio-Dorado-Manzanares M, Diaz-Pavon JM, Reyes-Diaz ML, Vazquez-Monchul JM, Garcia-Cabrera AM, et al. Learning curve in robotic rectal cancer surgery: current state of affairs. Int J Colorectal Dis 2016;31:1807-15.

29. Hojo K, Vernava AM 3rd, Sugihara K, Katumata K. Preservation of urine voiding and sexual function after rectal cancer surgery. Dis Colon Rectum 1991;34:532-9.

30. Park SY, Choi GS, Park JS, Kim HJ, Ryuk JP, Yun SH. Urinary and erectile function in men after total mesorectal excision by laparoscopic or robot-assisted methods for the treatment of rectal cancer: a case-matched comparison. World J Surg 2014;38:1834-42. 